\title{
On the analysis of heterogeneous fluids with jumps in the viscosity using a discontinuous pressure field
}

\author{
Sergio R. Idelsohn • Monica Mier-Torrecilla • \\ Norberto Nigro • Eugenio Oñate
}

Received: 17 June 2009 / Accepted: 11 November 2009 / Published online: 10 December 2009

(C) Springer-Verlag 2009

\begin{abstract}
Heterogeneous incompressible fluid flows with jumps in the viscous properties are solved with the particle finite element method using continuous and discontinuous pressure fields. We show the importance of using discontinuous pressure fields to avoid errors in the incompressibility condition near the interface.
\end{abstract}

Keywords Heterogeneous fluids · Multi-fluids ·

Multiphase flows · Incompressible Navier-Stokes equations · Free-surfaces · Interfaces

\section{Introduction}

The simultaneous presence of multiple fluids with varying properties in external or internal flows is found in daily life, in marine environmental problems, and numerous industrial processes, among many other practical situations. These types of flow are labeled "multi-fluid" or simply "heterogeneous fluids" and they typically exist in different forms depending on their phase distribution. Examples are gasliquid transport, magma chambers, fluid-fuel interactions, crude oil recovery, spray cans, sediment transport in rivers

S. R. Idelsohn is a ICREA Research Professor at CIMNE.

S. R. Idelsohn $(\varangle) \cdot$ M. Mier-Torrecilla $\cdot$ N. Nigro · E. Oñate International Centre for Numerical Methods in Engineering (CIMNE), Gran Capitan s/n, Technical University of Catalonia, 08034 Barcelona, Spain

e-mail: sergio@cimne.upc.edu

N. Nigro

Universidad Nacional del Litoral, Santa Fe, Argentina

E. Oñate

Universitat Politécnica de Cataluña, Barcelona, Spain and floods, pollutant transport in the atmosphere, cloud formation, fuel injection in engines, bubble column reactors and spray dryers for food processing, to name only a few. This demonstrates the large incidence and also importance of multi-fluid flows, which probably occur even more frequently than single phase flows [1].

As a result of the interaction between the different fluid components, multi-fluid flows are rather complex and very difficult to describe theoretically. Despite the practical importance of the problem and the intensive work carried out in the last decade for the development of suitable mathematical and computational models, it is widely accepted that the numerical study of heterogeneous flows is still a major challenge [1].

Computing the interface between two immiscible fluids or the free-surfaces is difficult because neither the shape nor the positions of the domains between the fluids are a priori known. In this kind of flows there are basically two approaches for computing interfaces, which are, using the terminology in [2], interface-tracking and interface capturing. The former computes the motion of the flow via a mixed Lagrangian-Eulerian approach [3] or a space-time approach $[4,5]$, where the numerical domain adapts itself to the shape and position of the interfaces. Standard interface-capturing methods consider both fluids as a single effective fluid with variable properties [6-8]. The interfaces are considered as a region of sudden change in the fluid properties. This approach requires an accurate modeling of the jump in the properties of the two fluids taking into account that the interfaces can move, bend and reconnect in arbitrary ways. Furthermore, prescribing exact boundary conditions in the interface is usually approximated. Non-standard interface-capturing methods have been developed to increase the accuracy in representing the interface, such as the Enhanced-Discretization Interface-Capturing Technique (EDICT) [9,10]. There 
are number of powerful techniques in the interface-tracking category, such as the DSD/SST formulation [4,5], which has been applied to a large number of two-fluid or free-surface flows, and the particle finite element method (PFEM) [11-13], which we focus on here.

In a previous publication [14] the authors solve the numerical problems of a density jump at the interfaces. In this paper the focus is on the numerical solution of a viscosity jump at the interface of two different fluids using the particle finite element method. The need for using a discontinuous pressure across an interface when there is surface tension was also recognized in applying the DSD/SST formulation to free-surface and two-fluid flows [5] and in several interface-capturing applications [15-20].

The layout of the paper is the following. In Sect. 2 the governing equations of heterogeneous fluids are presented together with the boundary and interfacial conditions. Section 3 deals with the main difficulties of the numerical solution of heterogeneous fluids and the techniques proposed to overcome them, which are tested in two numerical examples in Sect. 4.

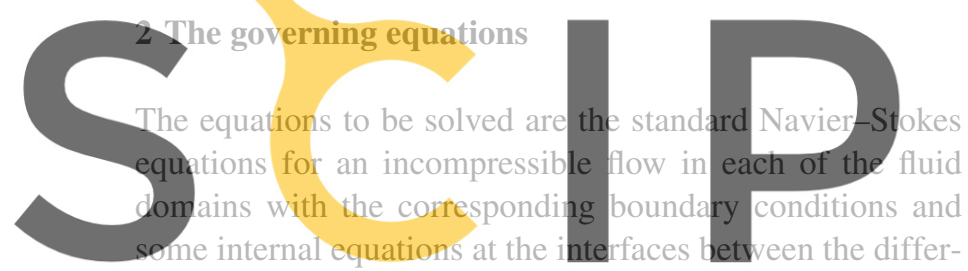
ent fluids.

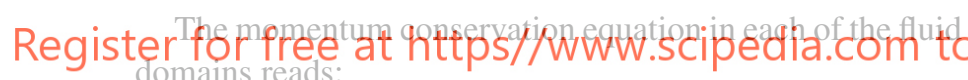

$\rho \frac{\mathrm{D} u_{i}}{\mathrm{D} t}=\frac{\partial \sigma_{i j}}{\partial x_{j}}+\rho f_{i}$

and the mass conservation:

$\frac{\mathrm{D} \rho}{\mathrm{D} t}+\rho \frac{\partial u_{i}}{\partial x_{i}}=0$

where $\rho$ is the density, $u_{i}$ are the Cartesian components of the velocity field, $\sigma_{i j}$ the Cauchy stress tensor, $f_{i}$ the source term (normally the gravity) and $\frac{\mathrm{D} \phi}{\mathrm{D} t}$ represents the total or material time derivative of a function $\phi$.

For heterogeneous materials $\rho$ is a function of the position $\rho=\rho(x)$. For incompressible flows, $\frac{\mathrm{D} \rho}{\mathrm{D} t}=0$. Nevertheless the spatial time derivative $\frac{\partial \rho}{\partial t}$ is not necessarily equal to zero $\left(\frac{\partial \rho}{\partial t} \neq 0\right)$. This is the reason why heterogeneous materials are more easily solved with Lagrangian formulations.

The constitutive equations for Newtonian fluids are:

$\sigma_{i j}=\tau_{i j}-p \delta_{i j}=\mu\left(\frac{\partial u_{i}}{\partial x_{j}}+\frac{\partial u_{j}}{\partial x_{i}}\right)-p \delta_{i j}$ where $\mu$ is the viscosity and $p$ the pressure assumed to be positive in compression.

Boundary and interface conditions

The standard boundary conditions for the Navier-Stokes equations are:

$\tau_{i j} n_{j}-p n_{i}=\bar{\sigma}_{n i}$ on $\Gamma_{\sigma}$;

$u_{i} n_{i}=\bar{u}_{n}$ on $\Gamma_{n}$;

$u_{i} t_{i}=\bar{u}_{t}$ on $\Gamma_{t}$;

where $n_{i}$ and $t_{i}$ are the components of the normal and tangential vectors to the boundary.

On the internal interfaces $\Gamma$ the conditions are:

$$
\begin{aligned}
& n_{i} \llbracket \sigma_{i j} \rrbracket n_{j}=\gamma \kappa \\
& t_{i} \llbracket \sigma_{i j} \rrbracket n_{j}=0 \\
& \llbracket u \rrbracket=0
\end{aligned}
$$

where $\llbracket \cdot \rrbracket$ represents the variable jump at each side of the interface, $\gamma$ is the surface tension coefficient, and $\kappa$ the curvature of the interface. These boundary conditions express that the velocities and the tangential stresses are continuous

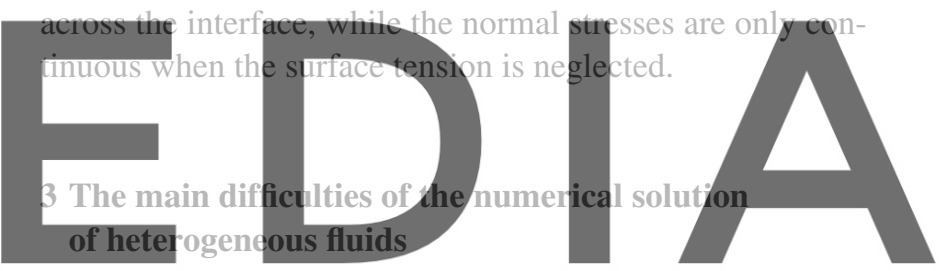

It is well known that one of the main advantages of many

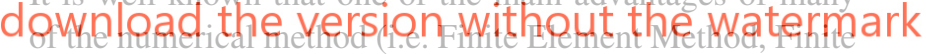

Volume Method, Finite Difference Method, etc) is the easy way to introduce a variation in the physical properties of the materials involved. For instance, each element in the Finite Element Method may have a different viscosity and this does not change the mathematical formulation of the method.

However, for immiscible incompressible flows, there are some particular facts than can introduce large errors in the simulation that make the results useless. These main difficulties are:

1. a correct definition of the interface position

2. stabilization errors at the interface where density jumps occur

3. pressure jumps at the interface where viscosity jumps are present

4. surface tension at immiscible interfaces

Some of these aspects and the corresponding numerical solution have been previously discussed by the authors in [14] and in many other references, e.g. [4, 16,21,22]. In particular many of the problems included in points 1 and 2 have 
been solved in [14]. For this reason, only a brief description of points 1 and 2 will be exposed here. The objective of this paper is to discuss more deeply points 3 and 4 .

\subsection{Correct definition of the interface position}

The main difference between an homogeneous fluid and an heterogeneous one is the presence of internal interfaces separating the different fluids. To know exactly where the interfaces are located is a key aspect for the accuracy of the algorithm to be employed. Two kinds of approaches may be used: (a) interface-tracking methods, in which the mesh follows the interfaces, and (b) interface-capturing methods (like level-set) in which the mesh is fixed in time and the interfaces move through the element domain. The first one has the advantage of providing an easy representation of interface jumps like pressure or pressure gradients. The main disadvantage is the need of frequent mesh updates. On the other hand, interface-capturing methods have difficulties in representing internal gradient jumps properly. In all the examples presented in this paper, interface-tracking techniques based on the particle finite element method will be used. For a more detailed description of the technique used to follow the inter-

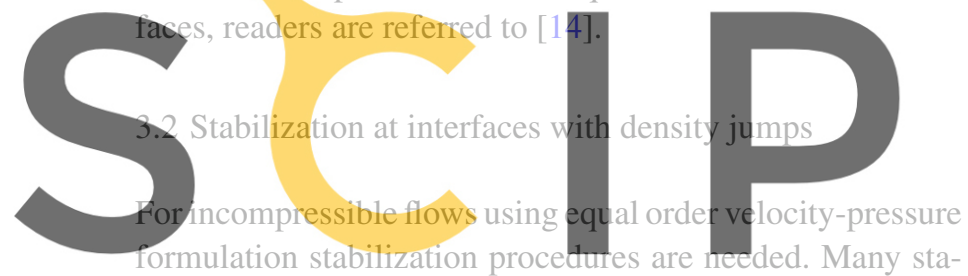
bilization procedures have been proposed in the literature.

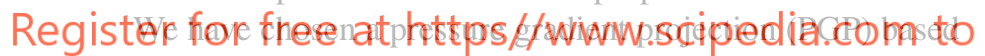
method $[23,24]$, where the pressure gradients are projected on a continuous field and the difference between the pressure gradients and their own projection is used as stabilization contribution:

$$
\frac{\partial}{\partial x_{i}} \tau\left(\frac{\partial p}{\partial x_{i}}-\pi_{i}\right)
$$

The term in brackets is interpreted as an approximation of the residual of the momentum equations and $\pi_{i}$ is a continuous function obtained from the projection of the pressure gradient on the velocity field and $\tau$ is a stabilization parameter.

In order to take into account the jumps at the interfaces for the density, in [14] the $\pi_{i}$ functions are defined as projections of the pressure gradients divided by $\rho(x)$. Thus, the stabilization term becomes:

$$
\frac{\partial}{\partial x_{i}} \tau\left(\frac{\partial p}{\partial x_{i}}-\rho(x) \pi_{i}\right)
$$

Expression (7) has been proved to be a better PGP stabilization term for heterogeneous fluid with large jumps in the density properties.
3.3 Pressure discontinuity at interfaces with viscosity jumps

Incompressible fluids need the introduction of the pressure variable because the solution cannot be obtained only as a function of the velocity field. In order to improve the efficiency of the method, standard approximations work with $\mathcal{C}^{0}$ pressure functions which improve the stabilization terms with a minimum number of degrees of freedom [11]. However, pressure is a physical discontinuous function in heterogeneous fluids in which there are jumps in the viscosity parameter. The use of a continuous pressure field is an approximation that introduces errors in the incompressibility condition that in certain cases produces unacceptable results. One of the main goals of this paper is to show the importance of using a discontinuous pressure field instead of a continuous one at least at the interface level.

The value of the pressure jump at the interface between two different fluids has been derived in [25]:

$$
\llbracket p \rrbracket=2 \llbracket \mu \rrbracket \frac{\partial u_{n}}{\partial n}-\gamma \kappa
$$

Expression (8) shows that the jump in the pressure field is not only a consequence of the surface tension, but also occurs

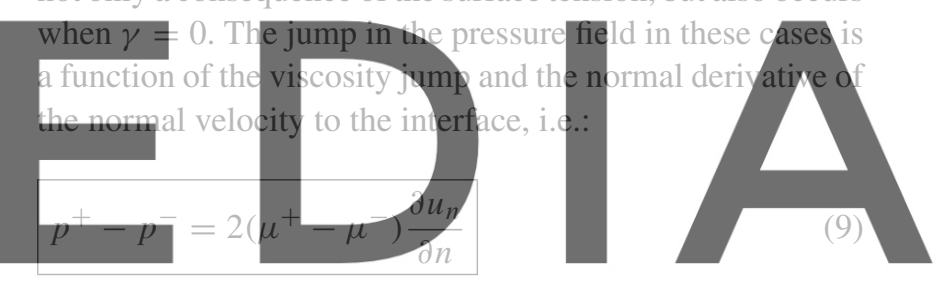

The main contribution of our work is to show with numerical downlqad the version with thut the watermark

discontinuity at interfaces with viscosity jumps, specially in terms of local mass conservation.

\subsection{Surface tension at immiscible interfaces}

The existence of two different fluids with different intermolecular attraction forces put in contact introduces the physical phenomena of surface tension. Surface tension is proportional to the curvature of the interface $\kappa$ and a parameter $\gamma$ that depends on the materials involved and has to be measured experimentally.

Surface tension effects are normally added in the numerical simulation as concentrated forces $f_{i}$ normal to the interface in the momentum equation:

$f_{i} n_{i}=-\gamma \kappa$

and, as mentioned before, they induce the following jump in the pressure field:

$p^{+}-p^{-}=-\gamma \kappa$ 
The importance of a correct simulation of the pressure jumps will be illustrated in the numerical examples presented in the next section.

\section{Numerical examples}

One of the questions that arises about the above theoretical results is why this discontinuous behavior of the pressure at the interface, in absence of surface tension, has not been usually taken into account. Pressure jumps have been previously treated connected with surface tension effects, see [15-20], and references therein. For example, a very common and well analyzed problem with a definite interface between fluids is the sloshing of a free surface separating water and air. Typically in this problem it is assumed that the pressure at the free surface is in equilibrium with the atmospheric pressure (the pressure in the air) and this assumption violates the results found above. The answer to this question is that being the viscosities of air and water so small, the pressure jump is also small, regardless the convective acceleration magnitude of the free surface, and therefore this jump is usually unnoticed. Fluid flow with different and high viscosities may be
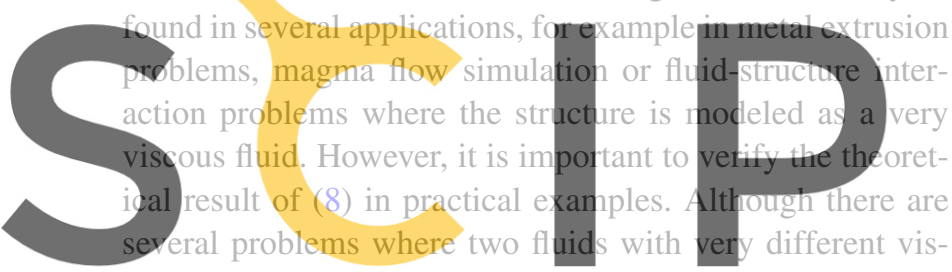

cosities are separated by an interface, it is not obvious to find an example where $\frac{\partial u_{n}}{\text { is relevant. In this section we propose }}$ Register for free at Mttes/dwwW. scipedia.com to

exist and that may serve as good tests to assess the ability of a given numerical method to represent the pressure discontinuity at the interface between two different fluids. Both examples consist in the extrusion against a wall of a rectangular domain composed by two fluids. In the first example the mesh is fixed and the solution stationary, while in the second example the mesh deforms in time with the domain. We will investigate the pressure solution and the volume conservation around the interface when continuous and discontinuous pressure discretizations are used. Table 1 shows an overview of the physical parameters taken in the simulations.

\subsection{Fixed-mesh example}

Figure 1 shows the definition of the first example. It deals with a rectangular 2D domain with a flow entering with velocity $\bar{u}_{x}=1 \mathrm{~m} / \mathrm{s}$ from the left boundary and flowing to the right where it finds an impermeable slip wall that deviates the flow upwards and downwards. The symmetry axis $(y=0)$ serves as a material curve separating fluid 1 from fluid 2 . Even though we have solved the problem with different cases of
Table 1 Overview of the examples

\begin{tabular}{llllrlrll}
\hline Example & Solution & Mesh & $\mu_{1}$ & $\mu_{2}$ & $\rho_{1}$ & $\rho_{2}$ & $\gamma$ & $\bar{u}_{x}$ \\
\hline 1. & Steady & Fixed & 5 & 1 & 1 & 1 & 0 & 1 \\
4.1 & Unsteady & Moving & 1 & 10 & 1 & 1 & 0 & 0.1 \\
4.2 & & & 1 & 10 & 1 & 1 & 0 & \\
& & & 1 & 1 & 1 & 1 & 3 & \\
4.3 & & & 1 & 10 & 1 & 10 & 5 & \\
\hline
\end{tabular}

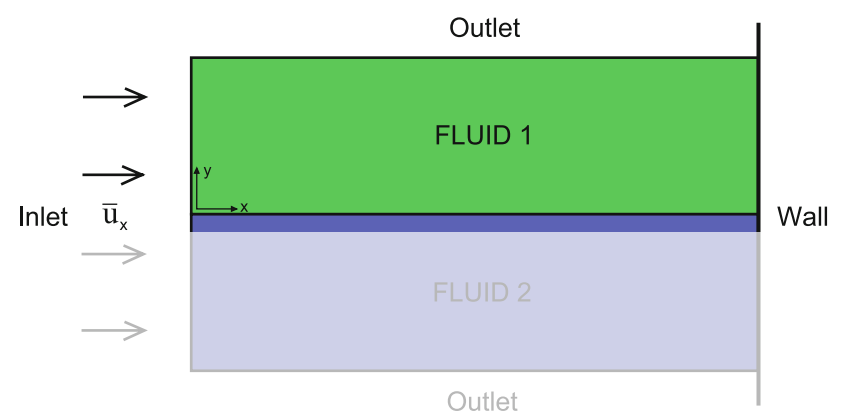

Fig. 1 Example definition

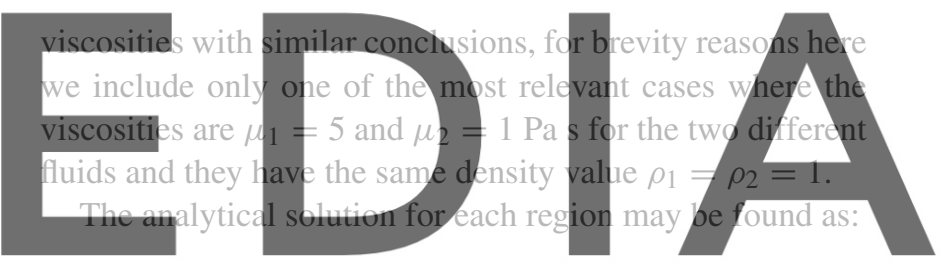

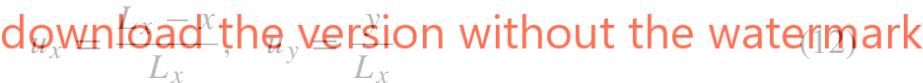

with $L_{x}=1 \mathrm{~m}$ the domain length in the $x$ direction and $L_{y}=0.5 \mathrm{~m}$ in the $y$ direction.

Then, $\frac{\partial u_{i}}{\partial x_{i}}=-\frac{1}{L_{x}}+\frac{1}{L_{x}}=0$.

In order to compute the pressure field using the momentum balance Eq. (1) we note that the acceleration is $\frac{\mathrm{D} u_{i}}{\mathrm{D} t}=$ $\frac{\partial u_{i}}{\partial t}+u_{j} \frac{\partial u_{i}}{\partial x_{j}}$ with

$\frac{\partial u_{i}}{\partial x_{j}} \Longrightarrow\left[\begin{array}{ll}\frac{\partial u_{x}}{\partial x} & \frac{\partial u_{x}}{\partial y} \\ \frac{\partial u_{y}}{\partial x} & \frac{\partial u_{y}}{\partial y}\end{array}\right]=\left[\begin{array}{cc}-\frac{1}{L_{x}} & 0 \\ 0 & \frac{1}{L_{x}}\end{array}\right]$

and

$u_{j} \frac{\partial u_{i}}{\partial x_{j}} \Longrightarrow\left[\begin{array}{c}\frac{x-L_{x}}{L_{x}^{2}} \\ \frac{y}{L_{x}^{2}}\end{array}\right]$ 
Therefore, the pressure gradient is such that compensates this force term,

$$
\begin{aligned}
& \frac{\partial u_{i}}{\partial t}+u_{j} \frac{\partial u_{i}}{\partial x_{j}}+\frac{1}{\rho} \frac{\partial p}{\partial x_{i}}=0, \quad \frac{\partial u_{i}}{\partial t}=0 \\
& \Longrightarrow \frac{\partial p}{\partial x}=-\rho \frac{x-L_{x}}{L_{x}^{2}}, \quad \frac{\partial p}{\partial y}=-\rho \frac{y}{L_{x}^{2}} \\
& \Longrightarrow p=p(x, y)=\frac{\rho}{L_{x}^{2}}\left(x L_{x}-\frac{1}{2}\left(x^{2}+y^{2}\right)\right)+C
\end{aligned}
$$

The above velocity vector field (12) with the pressure field (14) is a solution for the Navier-Stokes problem for each region: $\rho \frac{\mathrm{D} u_{i}}{\mathrm{D} t}=\frac{\partial}{\partial x_{j}} \tau_{i j}-\frac{\partial}{\partial x_{i}} p+\rho f_{i}$. Due to the fact that a linear velocity field is proposed as the steady solution of this problem, $\tau_{i j}$ is constant with no contribution on the right hand side.

Finally, we have to match both solutions at the interface and verify the stress equilibrium there. We found that the pressure at the interface is discontinuous, with a jump given by (8). For this problem the pressure jump is

$p^{+}-p^{-}=2\left(\mu^{+}-\mu^{-}\right) \frac{\partial u_{n}}{\partial n}=2\left(\mu_{1}-\mu_{2}\right) \frac{1}{L_{x}}$

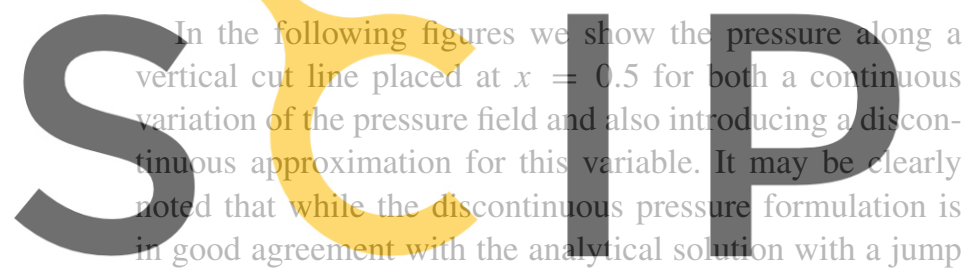

matching the theoretical expression, the continuous pressure

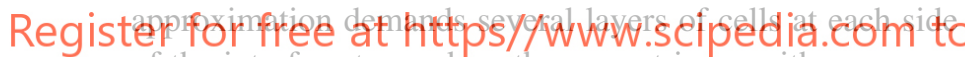
of the interface to produce the correct jump with an excessive diffusion (Fig. 2). In other vertical cuts along $x$-direction not included here, the pressure also presents an oscillatory behavior with a well defined undershoot and overshoot at the interface.

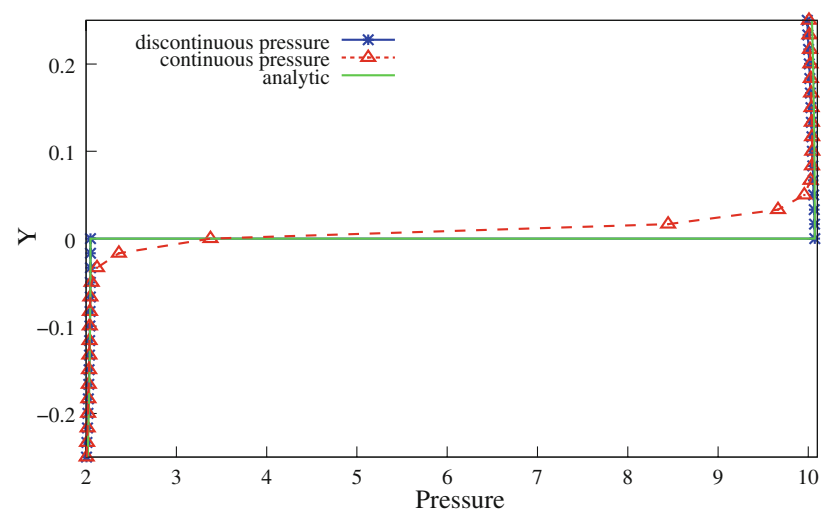

Fig. $2 \mu_{1}=5, \mu_{2}=1, \rho_{1}=\rho_{2}=1$. Pressure cut at $x=0.5$ for continuous versus discontinuous pressure approximations, compared with the analytic solution

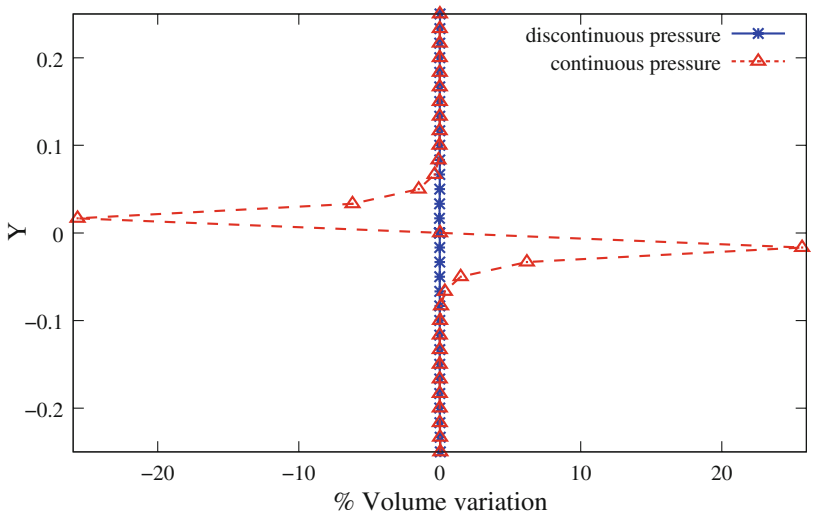

Fig. $3 \mu_{1}=5, \mu_{2}=1, \rho_{1}=\rho_{2}=1$. Volume variation at $x=0.5$ for continuous versus discontinuous pressure approximations

In Fig. 3 we plot the volume variations computed as the divergence of the velocity vector field to highlight the differences between using a continuous and a discontinuous pressure field. It is remarkable that there are at least two orders of magnitude between the volume variations in the vicinity of the interface in favor of the discontinuous treatment of the pressure field which practically give no volume

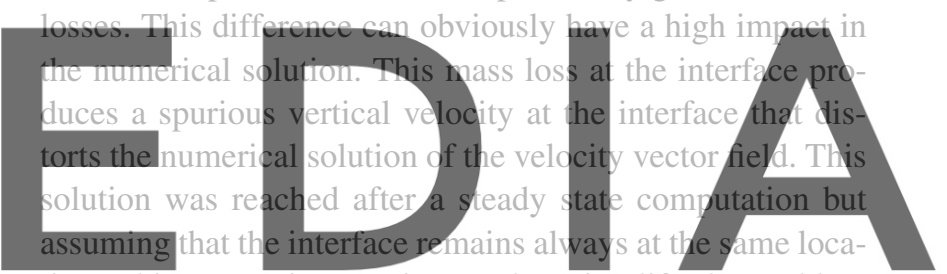
tion. This constraint was imposed to simplify the problem in order to see clearly the different response of a continu-

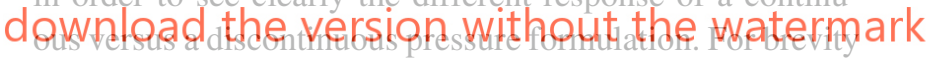

reasons we have not included here neither results with different viscosity values, nor the influence of different stabilization approaches. Nevertheless the conclusions are the same as before: 1. a sharp definition of the discontinuous pressure field, against a diffusive behavior of the pressure field requiring several cell layers to capture the pressure jump; and 2. a better fulfillment of the incompressibility constraint for the discontinuous pressure approach than for a continuous pressure formulation.

\subsection{Moving-mesh example}

Next we consider an example with moving-mesh, gravity and where the bottom free-surface is fixed $\left(u_{y}=0\right.$ at $\left.y=0\right)$, see Fig. 4. All walls are considered to be slip. Identical conclusions as in the previous example will be obtained concerning the pressure jump.

In this example, due to the choice of the physical parameters, viscous effects dominate over inertial ones, i.e. the quadratic pressure coming from the unsteady terms of the Navier-Stokes equations and described in the previous 
Fig. 4 Example settings and analytical pressure solution
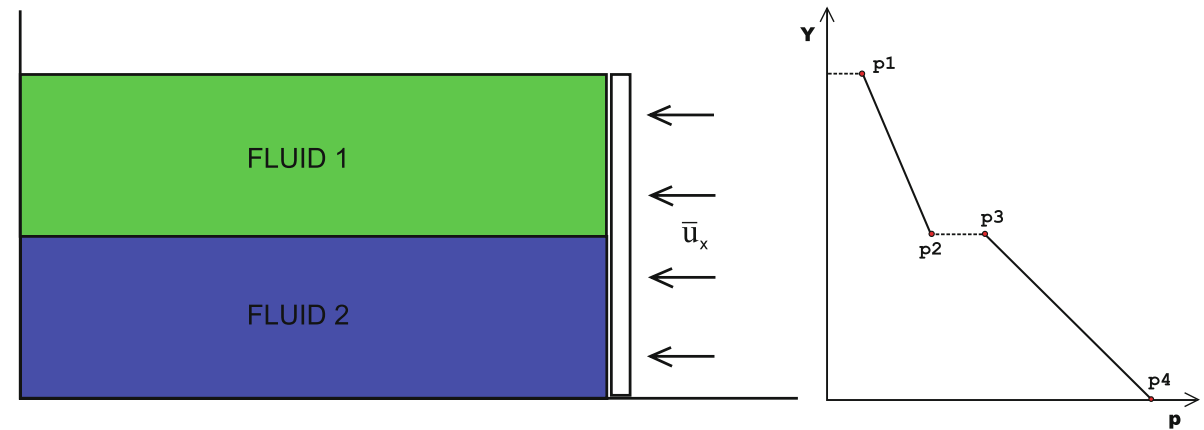

example is two orders of magnitude smaller than the "viscous" pressure and therefore can be neglected. Thus the analytical solution for this example is:

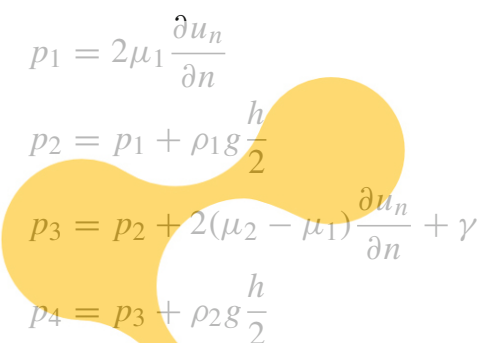

(16a)

(16b)

(16c)

$p_{4}=p_{3}+\rho_{2} g \frac{1}{2}$

$(16 d)$

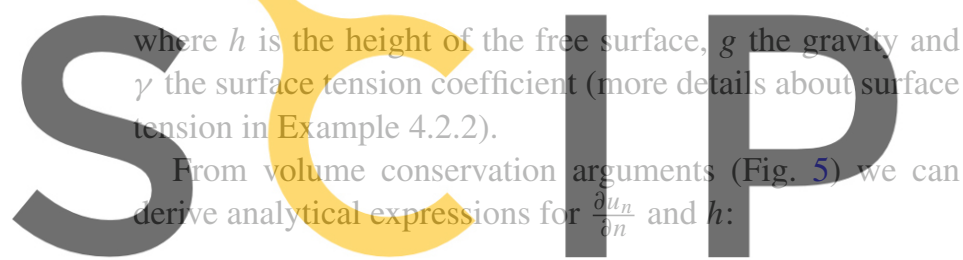

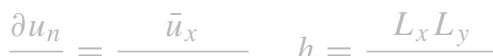

(17)

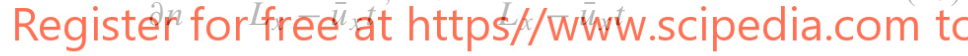

The piston speed has been set to $\bar{u}_{x}=0.1 \mathrm{~m} / \mathrm{s}$, the initial domain sizes to $L_{x}=0.8$ and $L_{y}=0.4$, and the gravity to $g=10 \mathrm{~m} / \mathrm{s}^{2}$.

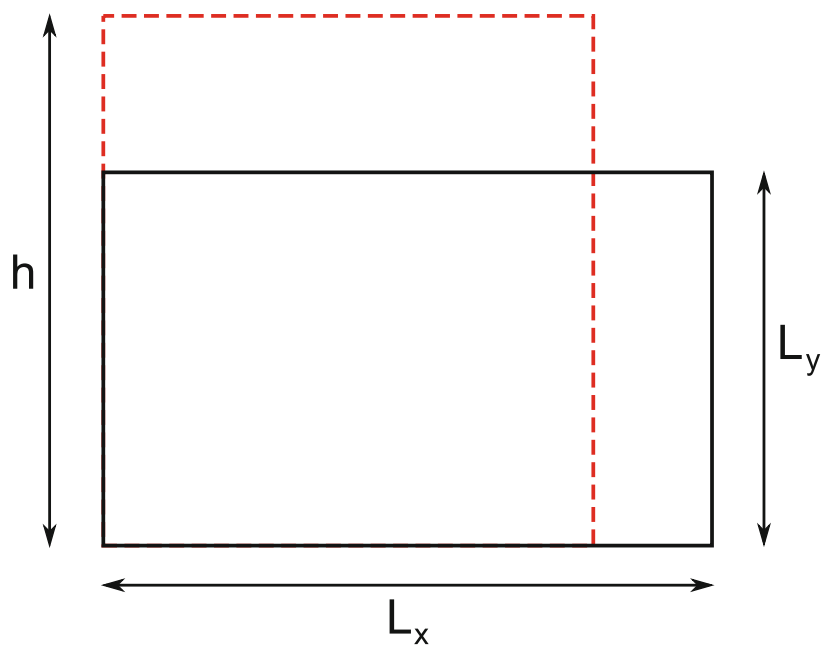

Fig. 5 Notation for the pressure solution
The following numerical tests will be solved to compare the solution using continuous and discontinuous pressure approximations:

1. Jump in the viscosity, with equal density and no surface tension

2. Equal density and viscosity, with surface tension

3. Jumps in the viscosity and density, including surface tension

In all these cases we will compare the pressure fields obtained and the volume variation of the incompressible fluid along a

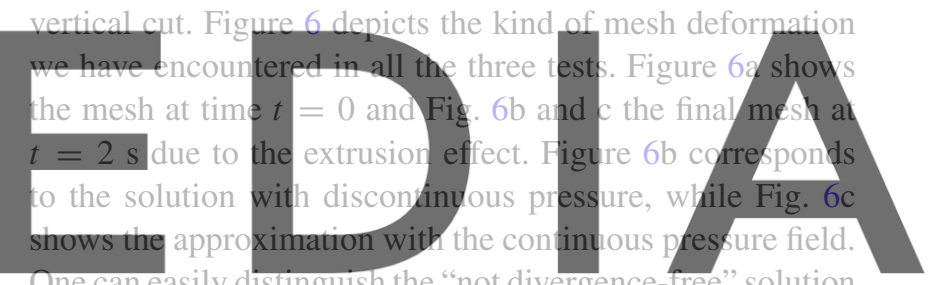

One can easily distinguish the "not divergence-free" solution

(Fig. 6c) close to the interface for the continuous pressure

dowkdoabithe version without the watermark

It is important to remark that linear shape functions for velocity and pressure $\left(\mathbb{P}_{1} / \mathbb{P}_{1}\right.$ elements) have been used for the calculations. In the discontinuous pressure results, as the interface is tracked with the moving mesh and lies at element edges, we have duplicated the pressure degrees of freedom at the interface nodes to allow for the pressure jump.

The volume variation we use to quantify $\partial u_{i} / \partial x_{i}$ in the following moving-mesh examples has been computed as $\varepsilon_{v}=\frac{V o l^{n}-V^{0} l^{0}}{V o l^{0}}$, where $V_{o l}{ }^{n}$ is the area associated to a node at time $n$ (Fig. 7).

Example 4.2.1 Jump in the viscosity, equal density, no surface tension

In this case the physical parameters used are: $\mu_{1}=1, \mu_{2}=$ $10, \rho_{1}=\rho_{2}=1, \gamma=0$. Figure 8 shows the good agreement of the discontinuous pressure solution against the exact value, while the continuous pressure approximation leads to an excessive diffusive behavior. Figure 9 plots the error in the volume conservation showing a similar conclusion as in the previous example. 
Fig. 6 Mesh deformation: a initial mesh, $t=0 \mathrm{~s}$; $\mathbf{b}$ mesh at $t=2 \mathrm{~s}$ and discontinuous pressure; c mesh at $t=2 \mathrm{~s}$ and continuous pressure showing a not divergence-free solution around the interface
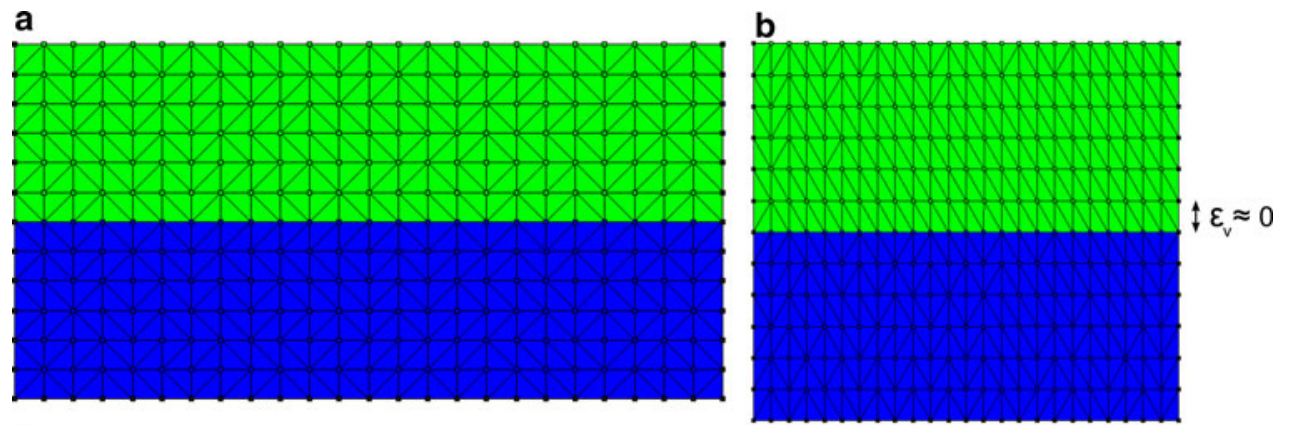

c

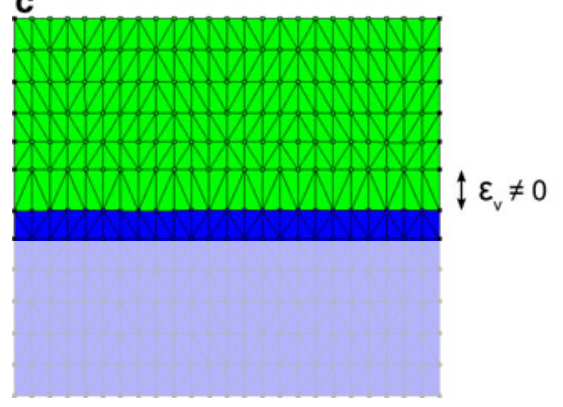

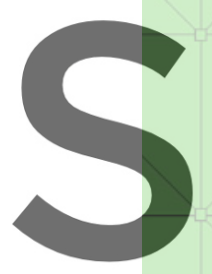
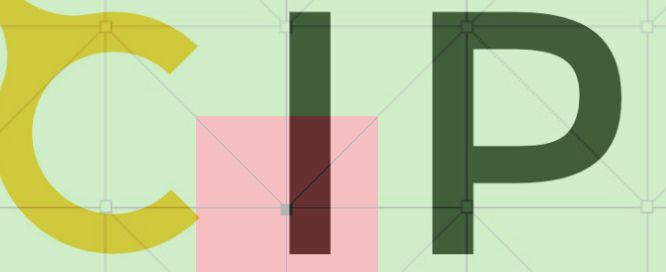

Register for free at https//www.scipedia.com to

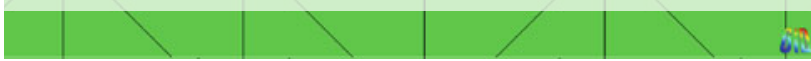

Fig. 7 Area associated to a node

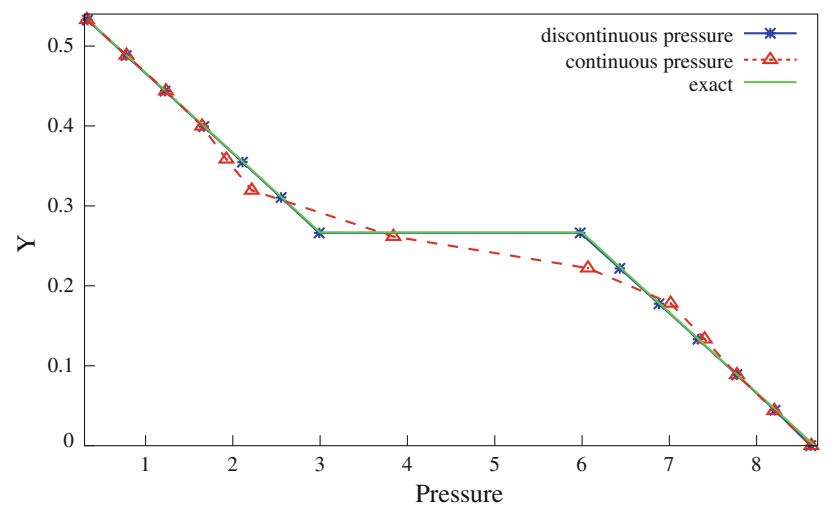

Fig. $8 \mu_{1}=1, \mu_{2}=10, \rho_{1}=\rho_{2}=1, \gamma=0$. Pressure cut at $t=2 \mathrm{~s}$ and $x=0.3$ for continuous versus discontinuous pressure approximations, compared with the exact solution

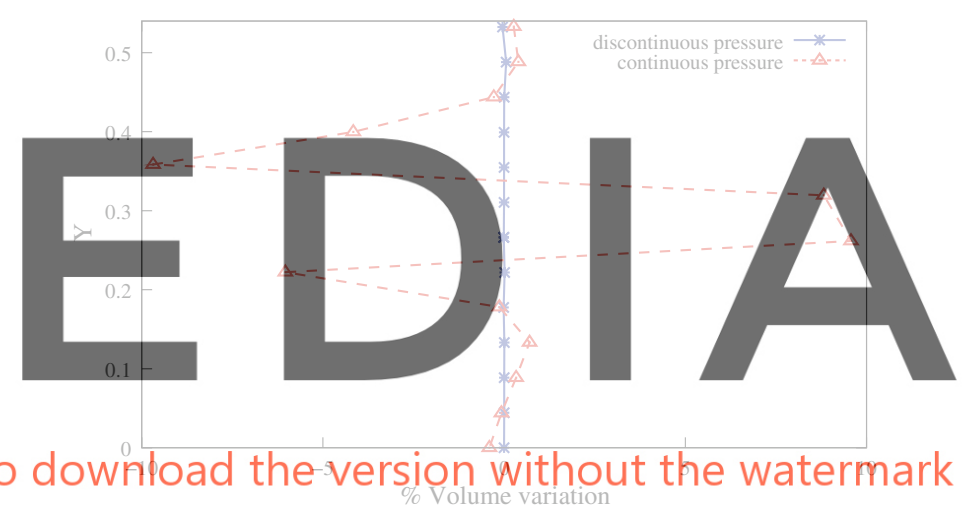

Fig. $9 \mu_{1}=1, \mu_{2}=10, \rho_{1}=\rho_{2}=1, \gamma=0$. Volume variation cut at $t=2 \mathrm{~s}$ and $x=0.3$ for continuous versus discontinuous pressure approximations

Example 4.2.2 No jumps in the density and viscosity but with surface tension

The weak form of the Navier-Stokes equations already includes the interfacial condition (5a) as a natural boundary condition in the case of no surface tension. In interfaces where the surface tension is present the following surface force must be computed: $f_{i} n_{i}=-\gamma \kappa$. In order to avoid the problems of evaluating the curvature and to have an analytical solution to compare the results with, we will consider a fictitious tension for planar surfaces [20]: $f_{i} n_{i}=-\gamma$. In this example the normal to the interface is taken as $\mathbf{n}=(0,-1)$.

In interface-capturing methods, the non-alignment of the interface with the mesh causes severe difficulties in the discretization of this localized surface tension force and spurious velocities appear if pressure is not allowed to be discon- 

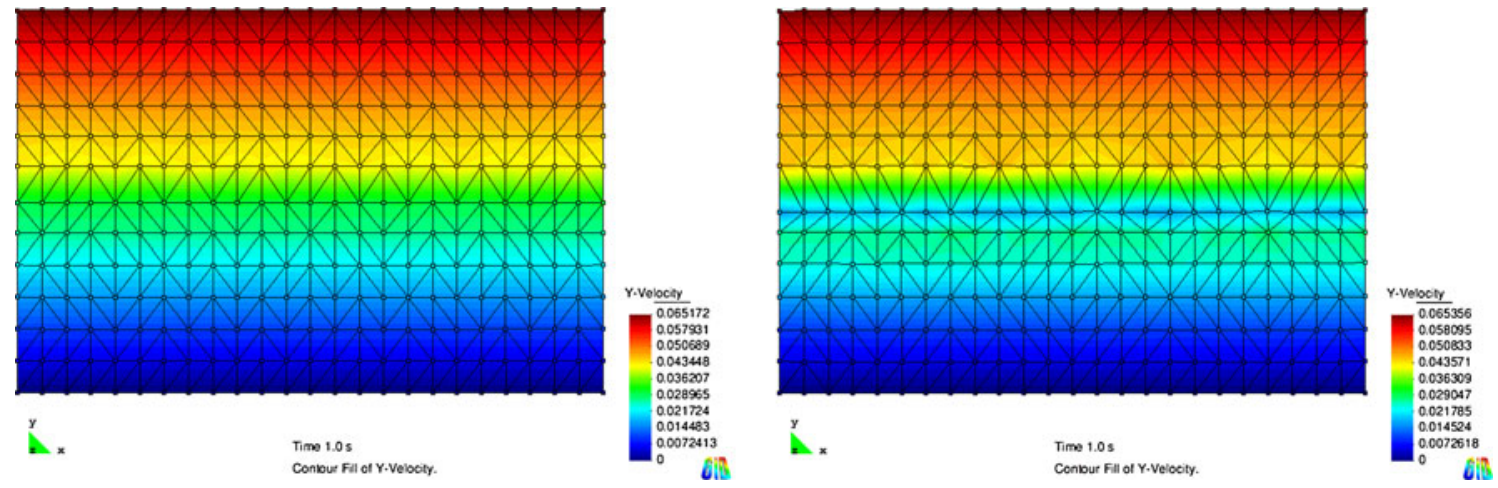

Fig. 10 Vertical velocity field at $t=1$ s for continuous pressure approximation. a Case with viscosity jump. b Case with surface tension. a $\mu_{1}=1, \mu_{2}=10, \rho_{1}=\rho_{2}=1, \gamma=0 . \mathbf{b} \mu_{1}=\mu_{2}=1, \rho_{1}=\rho_{2}=1, \gamma=3$

tinuous inside the elements cut by the interface $[19,20,26]$. The same spurious velocities appear in interface-tracking methods when pressure is approximated continuously, as shown in Fig. 10b.

In order to investigate the influence in the velocity field of the pressure jump due to different viscosities and to the surface tension, we have set the viscosity, the density and the surface tension force to have an equal jump. We know that
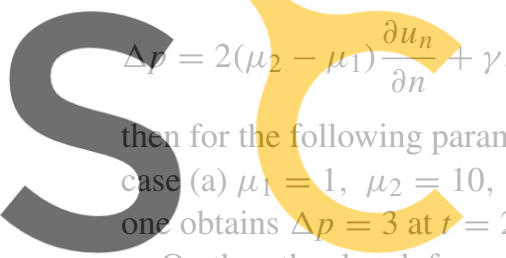

for the following parameters

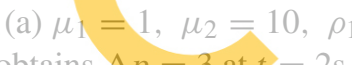

obtains $\Delta p=3$ at $t=2 \mathrm{~s}$

On the other hand, for
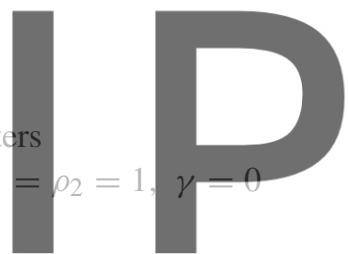

case (b) $\mu_{1}=\mu_{2}=1, \rho_{1}=\rho_{2}=1, \gamma=3$

Register for free at Bittps//www.scipedia.com to

Figure $10 \mathrm{a}$ and $\mathrm{b}$ shows the velocity results for the continuous pressure approximation. In both cases the pressure profiles are quite similar (Fig. 11), but in the case with surface tension, the non-physical velocities lead to much larger volume variations (Fig. 12). All these difficulties are avoided using a discontinuous pressure approximation (case (a) in Example 4.2.1 and Figs. 8 and 9, and case (b) in Figs. 11 and 12).

\section{Example 4.2.3 Jumps in the viscosity and density, including surface tension}

A density jump at the interface does not introduce a jump in the pressure field but in the pressure gradient. In this case accurate results can only be achieved with a discontinuous pressure gradient in the stabilization term at the interface [14] as explained in Sect. 3.2.

In the following example, we consider the case where a jump in both the pressure field and also in the pressure gradient is needed to obtain accurate results. We introduce now a jump in the viscosity, in the density and also surface tension:

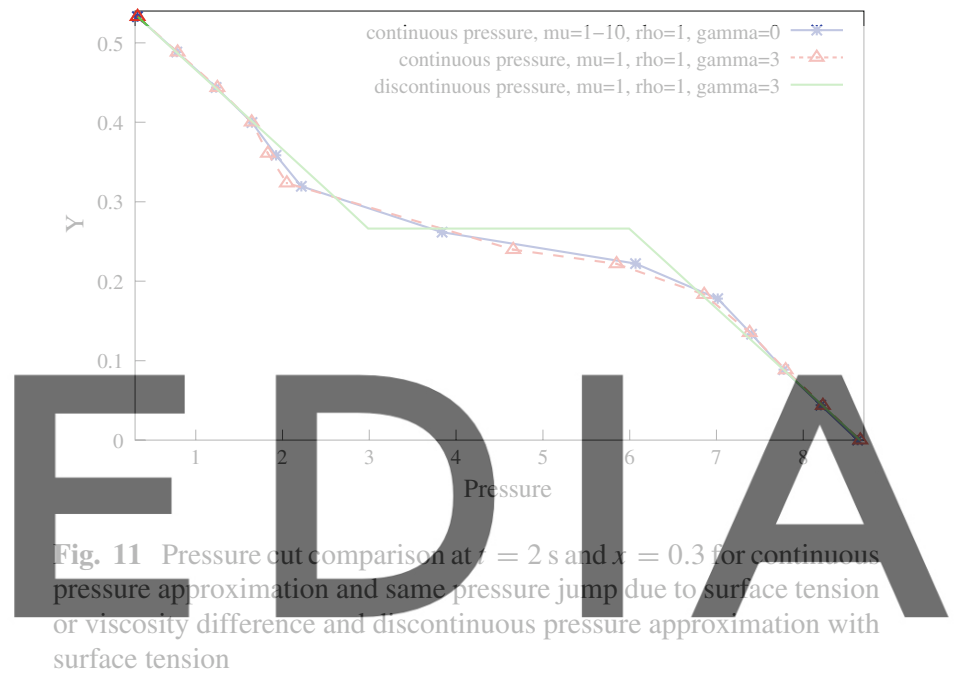

download the version without the watermark

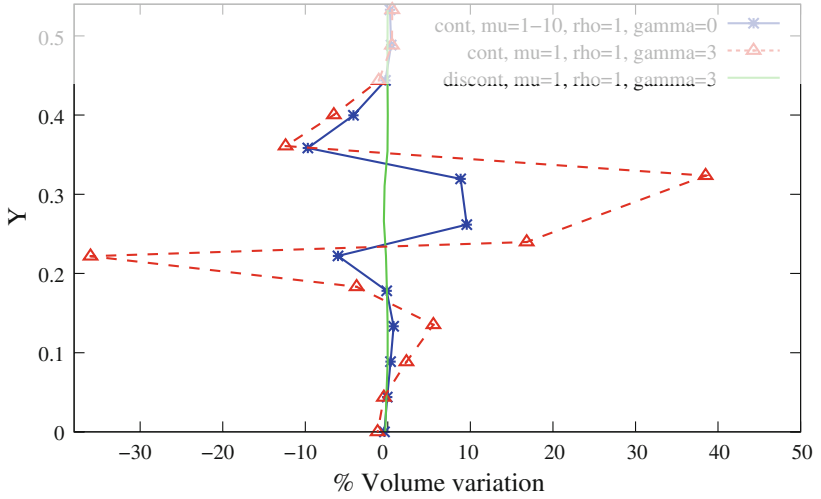

Fig. 12 Volume variation comparison at $t=2 \mathrm{~s}$ and $x=0.3$ for continuous pressure approximation and same pressure jump due to surface tension or viscosity difference and discontinuous pressure approximation with surface tension

$\mu_{1}=1, \mu_{2}=10, \rho_{1}=1, \rho_{2}=10, \gamma=5$. Figures 13 and 14 respectively show the pressure profile and the volume variation along $x=0.3$ at time $t=2 \mathrm{~s}$. The continuous pressure solution shows a volume variation over $15 \%$ while 


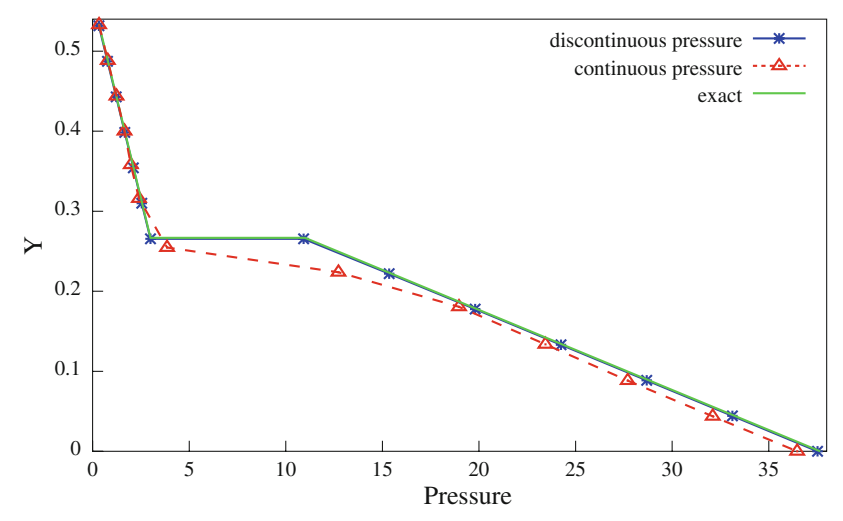

Fig. $13 \mu_{1}=1, \mu_{2}=10, \rho_{1}=1, \rho_{2}=10, \gamma=5$. Pressure cut at $t=2 \mathrm{~s}$ and $x=0.3$ for continuous versus discontinuous pressure approximations, compared with the exact solution

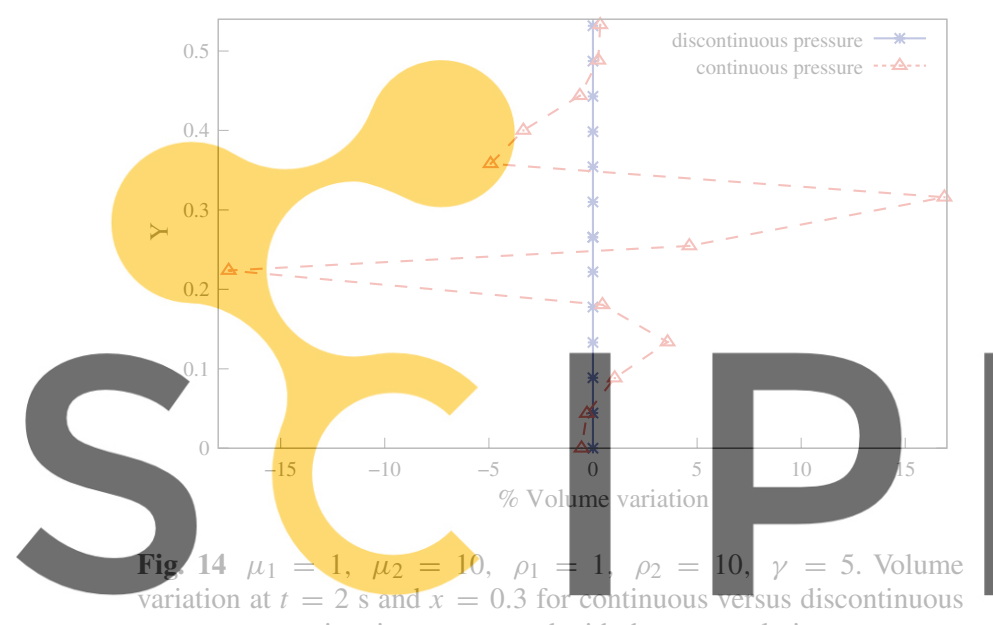

pressure approximations, compared with the exact solution

Register for free at https//www.scipedia.com to

in the discontinuous solution the variation is almost zero, as the divergence-free condition requires.

\section{Conclusions}

Incompressible fluid flows cannot be solved only as a function of the velocity field. They need the introduction of the pressure field as main unknown. This pressure field must satisfy the Babuska-Brezzi condition in order to avoid pressure oscillations. The most efficient way is to introduce stabilized continuous pressure fields of the same order of approximation as the velocity field. Nevertheless, continuous pressure fields are sometimes non-physical. This is the case for heterogeneous fluids with jumps in the viscous properties or surface tension. In these cases the introduction of pressure fields with discontinuity at the interface is essential to avoid large volume variations at the interface level with a noticeable imbalance of the incompressibility constraint. The magnitude of the pressure jump is proportional to the viscous jump multiplied by the variation of the normal velocity to the inter- face. Often this jump is small, and perhaps absorbed in the approximation error. However, in some physical problems like extrusion of materials where both the viscosity jump and the convective acceleration may be important this pressure jump must be modeled in order to avoid unacceptable volume variations. Other situations, like fluid-structure interactions where the solid is modeled as a fluid with a large viscosity and density, justify the use of a discontinuous pressure approximation. Unbalanced volume variations larger than $20 \%$ have been observed in the examples presented when the standard continuous pressure field is used.

Acknowledgments M. Mier-Torrecilla thanks the Catalan Agency for Administration of University and Research Grants (AGAUR), the European Social Fund and CIMNE for their support. N. Nigro thanks the financial support received from "Consejo Nacional de Investigaciones Cientificas y Tecnicas" and "Universidad Nacional del Litoral". He also thanks the International Center for Numerical Methods in Engineering for inviting him to Barcelona to work in this topic.

\section{References}

1. Sommerfeld M, van Wachem B, Oliemans R (eds) (2007) ERCOF-

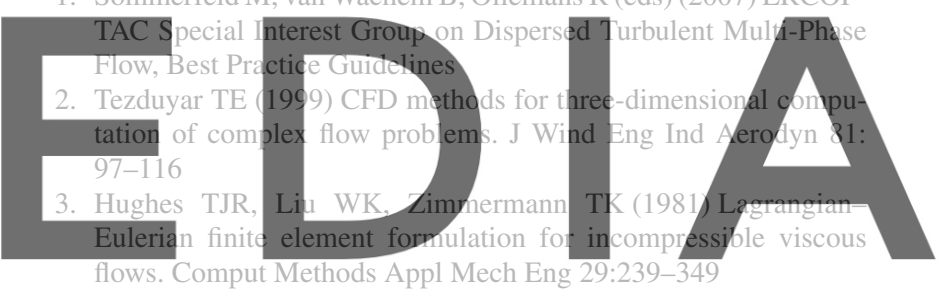

4. Tezduyar TE, Behr M, Liou J (1992) A new strategy for finite ele-

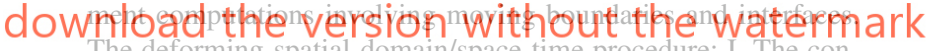
cept and preliminary numerical tests. Comput Methods Appl Mech Eng 94:339-351

5. Tezduyar TE, Behr M, Mittal S, Liou J (1992) A new strategy for finite element computations involving moving boundaries and interfaces. The deforming-spatial-domain/space-time procedure: II. Computation of free-surface flows, two-liquid flows and flows with drifting cylinders. Comput Methods Appl Mech Eng 94: 353-371

6. Sethian JA (2001) Evolution, implementation, and application of level set and fast marching methods for advancing fronts. J Comput Phys 169:503-555

7. Osher S, Fedkiw RP (2001) Level set methods: an overview and some recent results. J Comput Phys 169:463-502

8. Guermond JL, Quartapelle L (2000) A projection FEM for variable density incompressible flows. J Comput Phys 165:167-188

9. Tezduyar T, Aliabadi S, Behr M (1998) Enhanced-discretization interface-capturing technique (EDICT) for computation of unsteady flows with interfaces. Comput Methods Appl Mech Eng 155:235-248

10. Tezduyar TE, Aliabadi S (2000) EDICT for 3D computation of two-fluid interfaces. Comput Methods Appl Mech Eng 190: 403-410

11. Idelsohn SR, Oñate E, Del Pin F (2004) The particle finite element method: a powerful tool to solve incompressible flows with freesurfaces and breaking waves. Int J Numer Methods Eng 61(7): 964-989 
12. Oñate E, Idelsohn SR, del Pin F, Aubry R (2004) The particle finite element method: an overview. Int J Comput Methods 1(2):267-307

13. Idelsohn SR, Oñate E, Del Pin F, Calvo N (2006) Fluid-structure interaction using the particle finite element method. Comput Methods Appl Mech Eng 195(17-18):2100-2123

14. Idelsohn SR, Mier-Torrecilla M, Oñate E (2009) Multi-fluid flows with the particle finite element method. Comput Methods Appl Mech Eng 198:2750-2767

15. Unverdi SO, Tryggvason G (1992) A front-tracking method for viscous, incompressible, multi-fluid flows. J Comput Phys 100: $25-37$

16. Scardovelli R, Zaleski S (1999) Direct numerical simulation of free-surface and interfacial flow. Annu Rev Fluid Mech 31: 567-603

17. Smolianski A (2005) Finite-Element/Level-Set/Operator-Splitting (FELSOS) approach for computing two-fluid unsteady flows with free moving interfaces. Int J Numer Methods Fluids 48:231-269

18. Li Z, Lubkin S (2001) Numerical analysis of interfacial twodimensional Stokes flow with discontinuous viscosity and variable surface tension. Int J Numer Methods Fluids 37:525-540

19. Minev PD, Chen T, Nandakumar K (2003) A finite element technique for multifluid incompressible flow using Eulerian grids. J Comput Phys 187:255-273
20. Gross S, Reusken A (2007) An extended pressure finite element space for two-phase incompressible flows with surface tension. J Comput Phys 224:40-58

21. Hyman JM (1984) Numerical methods for tracking interfaces. Phys D Nonlinear Phenom 12:396-407

22. Floryan JM, Rasmussen H (1989) Numerical methods for viscous flows with moving boundaries. Appl Mech Rev 42:323-337

23. Codina R, Blasco J (2000) Stabilized finite element method for the transient Navier-Stokes equations based on a pressure gradient projection. Comput Methods Appl Mech Eng 182:277-300

24. Oñate $\mathrm{E}$ (2000) A stabilized finite element method for incompressible viscous flows using a finite increment calculus formulation. Comput Methods Appl Mech Eng 182:355-370

25. Kang M, Fedkiw RP, Liu XD (2000) A boundary condition capturing method for multiphase incompressible flow. J Sci Comput 15:323-360

26. Ganesan S, Matthies G, Tobiska L (2007) On spurious velocities in incompressible flow problem with interfaces. Comput Methods Appl Mech Eng 196:1193-1202
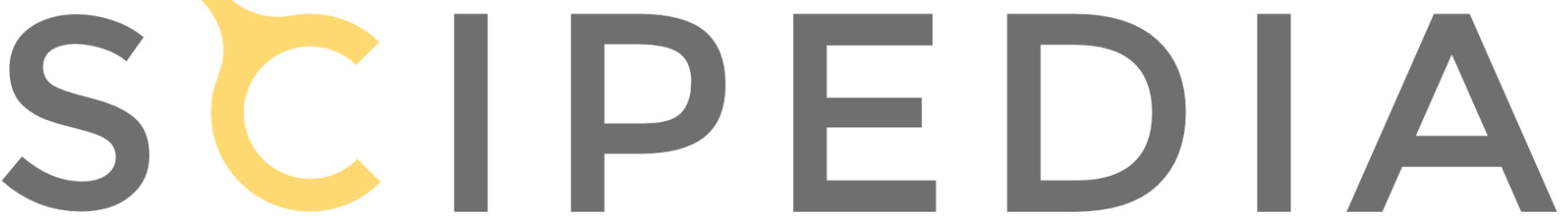

Register for free at https//www.scipedia.com to download the version without the watermark 